\title{
The near-infrared and ice-band variability of Haro 6-10
}

\author{
Ch. Leinert ${ }^{1}$, T. L. Beck ${ }^{2,3}$, S. Ligori ${ }^{1}$, M. Simon ${ }^{2,3}$, J. Woitas ${ }^{1}$, and R. R. Howell ${ }^{4, \star}$ \\ 1 Max-Planck-Institut für Astronomie, Königstuhl 17, 69117 Heidelberg, Germany \\ 2 State University of New York at Stony Brook, Stony Brook, NY 11974-3800, USA \\ 3 Visiting Astronomer at the Infrared Telescope Facility, which is operated by the University of Hawaii under \\ contract to the National Aeronautics and Space Administration \\ ${ }^{4}$ University of Wyoming, Laramie, WY 82071, USA
}

Received 1 December 2000 / Accepted 19 January 2001

\begin{abstract}
We have monitored the angularly resolved near infrared and $3.1 \mu \mathrm{m}$ ice-band flux of the components of the young binary Haro 6-10 on 23 occasions during the years 1988 to 2000. Our observations reveal that both the visible star Haro 6-10 (Haro 6-10S) and its infrared companion (Haro 6-10N) show significant variation in flux on time scales as short as a month. The substantial flux decrease of Haro 6-10S over the last four years carries the reddening signature of increased extinction. However, a comparable $K$-band flux increase observed in the IRC is associated with a dimming in the $H$-band and cannot be explained by lower extinction. Absorption in the $3.1 \mu \mathrm{m}$ water-ice feature was always greater towards the IRC during our observations, indicating a larger amount of obscuring material along its line of sight. We detect variability in the ice-band absorption towards Haro 6-10S and Haro $6-10 \mathrm{~N}$, significant at the $3.5 \sigma$ and $2.0 \sigma$ levels, respectively.
\end{abstract}

Key words. stars: binaries: close - infrared: stars - stars: individual: Haro-10 - stars: pre-main sequence - stars: variables: general

\section{Introduction}

Haro 6-10 is a prominent member of the small class of young binaries with infrared companions (Koresko et al. 1997, hereafter K97). Infrared companions (IRCs) to $\mathrm{T}$ Tauri stars are characterized by faintness or nondetection in the visible, very red spectral energy distributions (SEDs), and strong variability in the infrared. The binary system Haro 6-10 consists of Haro 6-10S, a K3-5 star (Goodrich 1986) seen in visible light through $A_{V} \approx 5.6$ mag extinction, and Haro $6-10 \mathrm{~N}$, the IRC, $1^{\prime \prime} 2$ to the north (Leinert \& Haas 1989a). Observations of the unresolved system show that its near infrared flux can vary significantly on timescales of months (Elias 1978; Leinert et al. 1996).

The infrared excess of Haro 6-10 is very pronounced compared to other $\mathrm{T}$ Tauri stars. Its unresolved SED is flat or rising between 1 and $100 \mu \mathrm{m}$, making Haro 6-10 a class I or "protostellar" T Tauri star in the system of Lada (1987). Haro 6-10 is unique among the infrared companion systems because angularly resolved observations

Send offprint requests to: Ch. Leinert, e-mail: leinert@mpia-hd.mpg.de

* Based in part on data obtained on the Calar Alto $3.5 \mathrm{~m}$, the IRTF, UKIRT, WIRO, TIRGO, and the La Palma Herschel telescopes. indicate that both components have this classification (K97). The IRC, however, dominates the flux of the system at wavelengths longward of $3.5 \mu \mathrm{m}$ and has an integrated luminosity twice that of Haro 6-10S (K97).

Angularly resolved observations of the $10 \mu \mathrm{m}$ silicate absorption feature indicate stronger extinction along the line of sight to the IRC (van Cleve et al. 1994). Whittet et al. (1985) list the unresolved Haro 6-10 system among the young stellar sources showing a deep $3.1 \mu \mathrm{m}$ waterice absorption feature. Angularly resolved observations revealed that the ice-band absorption was stronger towards the IRC (Leinert et al. 1996). Apparently the lines of sight to Haro 6-10S and its IRC contain very different amounts of absorbing material even though their projected separation on the sky is only $\approx 170$ AU.

Observations made to date have not successfully explained the cause of the photometric variability and extinction observed in the Haro 6-10 system. K97 proposed that IRCs are otherwise normal young stars experiencing strong, episodic extinction and variable accretion perhaps stimulated by the orbital motion of the binary. We have monitored the near infrared flux and ice-band absorption of both components of Haro 6-10 at every opportunity. Our observations provide new insights to the variability of Haro 6-10 and its IRC. 
Table 1. Journal of observations

\begin{tabular}{|c|c|c|c|c|c|}
\hline Date & Type & Result & Telescope & Instrument & Reference \\
\hline 22 Sep. 1986 & 1D speckle & $K_{\mathrm{sys}}, L_{\mathrm{sys}}^{\prime}$ & Calar Alto & own, InSb & 1 \\
\hline 11 Sep. 1987 & 1D speckle & $H_{\text {sys }}$ & Calar Alto & own, InSb & 1 \\
\hline 25-27 Sep. 1988 & 1D speckle & $H, K, L^{\prime}, M_{\mathrm{s}}$ & Calar Alto & own, InSb & 1 \\
\hline 11/16 Oct. 1989 & 1D speckle & $K$, ice, dust, $L^{\prime}$ & Calar Alto & own, InSb & 2 \\
\hline 19-27 Sep. 1991 & 1D speckle & $H, K$, ice, dust, $L_{\mathrm{n}}, L^{\prime}$ & Calar Alto & own, InSb & 2 \\
\hline 28 Sep. 1991 & lunar occultation & $K$ & La Palma & special, InSb & 3 \\
\hline 29 Oct. 1991 & 2D speckle & $H, K$, ice, dust, $L^{\prime}$ & Calar Alto & $1-5 \mu \mathrm{m}$ camera & 2 \\
\hline 06-10 Jan. 1993 & 1D speckle & $H, K$, ice, dust, $L_{\mathrm{n}}, L^{\prime}, M_{\mathrm{s}}$ & Calar Alto & own, InSb & 2 \\
\hline 28 Jan. 1994 & 2D speckle & $K$ & Calar Alto & Black MAGIC & \\
\hline 15 Dec. 1994 & 2D speckle & $K$ & Calar Alto & Blue MAGIC & \\
\hline 08 Oct. 1995 & 2D speckle & $K$ & Calar Alto & Blue MAGIC & \\
\hline 26 Mar. 1996 & 1D speckle & K & Calar Alto & own, InSb & \\
\hline 27 Sep. 1996 & 2D speckle & $K$ & Calar Alto & Blue MAGIC & \\
\hline 30 Sep. 1996 & 1D speckle & $K$, ice, dust, $L_{\mathrm{n}}$ & Calar Alto & own, InSb & \\
\hline 14/15 Nov. 1997 & 1D speckle & $H, K$, ice, dust, $L_{\mathrm{n}}, L^{\prime}, M_{\mathrm{s}}$ & Calar Alto & own, InSb & \\
\hline 06 Mar. 1998 & 2D Speckle & $K, L^{\prime}$ & IRTF & NSFCam & \\
\hline 14 Sep. 1998 & Imaging & $J_{\mathrm{sys}}, H, K, L^{\prime}$ & IRTF & NSFCam & \\
\hline 10 Dec. 1998 & Imaging & $J_{\text {sys }}, H, K, C V F$ filters ${ }^{*}, L^{\prime}$ & IRTF & NSFCam & \\
\hline 02 Sep. 1999 & 2D speckle & $J_{\text {sys }}, H_{\text {sys }}, K_{\text {sys }}$ & Calar Alto & $\Omega_{\text {Cass }}$ & \\
\hline 18 Sep. 1999 & Imaging & $H_{\mathrm{sys}}, K, L^{\prime}, M^{\prime \prime}$ & WIRO & IoCam 1 & \\
\hline 08 Oct. 1999 & Imaging & $H_{\mathrm{sys}}, K, L^{\prime}, M^{\prime \prime}$ & WIRO & IoCam 1 & \\
\hline 03 Nov. 1999 & Imaging & $K, C V F$ filters ${ }^{*}, L^{\prime}$ & IRTF & NSFCam & \\
\hline 06 Dec. 1999 & Imaging & $J_{\text {sys }}, H, K$, ice, dust, $L^{\prime}, M_{\mathrm{s}}$ & UKIRT & TUFTI & \\
\hline 26 Jan. 2000 & Imaging & $K, L^{\prime}$ & IRTF & NSFCam & \\
\hline 07 Mar. 2000 & Imaging & $K, C V F$ filters ${ }^{*}, L^{\prime}$ & IRTF & NSFCam & \\
\hline
\end{tabular}

${ }^{1}$ Leinert \& Haas (1989b).

${ }^{2}$ Leinert et al. (1996).

${ }^{3}$ Richichi et al. (1994).

${ }^{*}$ IRTF CVF filters are centered at 2.4, 2.9, 3.05 and $3.4 \mu \mathrm{m}$.

\section{Observations}

Our data (Table 1), consisting of a heterogenous set of observations obtained at different telescopes with different techniques, required standardisation in calibration. We based the calibration on the flux values for Vega given by Tokunaga (1999), and interpolated smoothly to the wavelengths of those filters not covered by his Table 7.5. The IRTF data were referenced to a magnitude scale in which Vega has zero magnitude at all wavelengths shortward of $20 \mu \mathrm{m}$, as given by Tokunaga. In this framework, the original flux calibration of the Calar Alto data was on a scale in which a star of magnitude of $0.06 \pm 0.01$ would have the Vega flux listed by Tokunaga. Magnitudes were determined using standards from the list of Elias et al. (1978). To have the same scale for both data sets, we adjusted the Calar Alto fluxes downwards by $6 \%$. The magnitudes are not affected by this recalibration. The overall consistency of the calibration of our data and the precision of our photometry should be better than $\pm 5 \%$.
For the speckle observations (Sects. 3.1 and 3.2), the calibration was applied to the total system flux. The fluxes of the components were obtained subsequently using their flux ratio derived from analysis of the speckle observations. For flux ratios smaller than 0.10, an additional uncertainty up to $10 \%$ enters the flux determination of the faint component, because the average error for the determination of the flux ratio in $H$ or $K$ is \pm 0.007 . Calibration of the imaging observations (in particular Sect. 3.3) was applied directly to the components of Haro 6-10.

\section{Results}

\subsection{Photometric variability}

Table 2 lists the near IR magnitudes and colors of Haro $6-10 \mathrm{~N}$ and $\mathrm{S}$. The IRC has been brighter than Haro 6-10S at $L^{\prime}$ and $M$ throughout our observations of the system. At shorter wavelengths, the IRC was significantly fainter than Haro 6-10S at the start of our observations and slowly 
Table 2. Individual magnitudes and colours for the components. A subscript $\mathrm{S}$ is for the visible star Haro 6-10S, and $\mathrm{N}$ is for the IRC, Haro 6-10N

\begin{tabular}{|c|c|c|c|c|c|c|c|c|c|c|c|c|c|}
\hline No. & Date & $H_{\mathrm{N}}$ & $H_{\mathrm{S}}$ & $K_{\mathrm{N}}$ & $K_{\mathrm{S}}$ & $H-K_{\mathrm{N}}$ & $H-K_{\mathrm{S}}$ & $L_{\mathrm{N}}^{\prime}$ & $L_{\mathrm{S}}^{\prime}$ & $K-L_{\mathrm{N}}^{\prime}$ & $K-L_{\mathrm{S}}^{\prime}$ & $M_{\mathrm{N}}$ & $M_{\mathrm{S}}$ \\
\hline 1 & 25-27 Sep. 1988 & 11.88 & 8.99 & 9.92 & 7.70 & 1.96 & 1.29 & 6.49 & 6.18 & 3.43 & 1.52 & 5.02 & 5.26 \\
\hline 2 & 11/16 Oct. 1989 & - & - & 9.76 & 7.30 & - & - & 6.64 & 5.73 & 3.12 & 1.63 & - & - \\
\hline 3 & 19-27 Sep. 1991 & 11.73 & 8.54 & 10.42 & 7.42 & 1.31 & 1.12 & 6.87 & 5.98 & 3.55 & 1.46 & - & - \\
\hline 4 & 28 Sep. 1991 & - & - & 9.60 & 7.10 & - & - & - & - & - & - & - & - \\
\hline 5 & 29 Oct. 1991 & 11.82 & 8.50 & 10.34 & 7.39 & 1.48 & 1.11 & 7.13 & 5.67 & 3.20 & 1.72 & - & - \\
\hline 6 & 06-10 Jan. 1993 & 12.55 & 8.63 & 9.84 & 7.34 & 2.71 & 1.29 & 6.35 & 5.85 & 3.49 & 1.49 & 4.82 & 4.95 \\
\hline 7 & 28 Jan. 1994 & - & - & 11.00 & 7.30 & - & - & - & - & - & - & - & - \\
\hline 8 & 15 Dec. 1994 & - & - & 9.54 & 7.21 & - & - & - & - & - & - & - & - \\
\hline 9 & 08 Oct. 1995 & - & - & 10.24 & 7.09 & - & - & - & - & - & - & - & - \\
\hline 10 & 26 Mar. 1996 & - & - & 9.82 & 7.18 & - & - & - & - & - & - & - & - \\
\hline 11 & 27 Sep. 1996 & - & - & 10.32 & 7.54 & - & - & - & - & - & - & - & - \\
\hline 12 & 30 Sep. 1996 & - & - & 9.91 & 7.39 & - & - & - & - & - & - & - & - \\
\hline 13 & 14/15 Nov. 1997 & 12.62 & 9.82 & 10.19 & 8.05 & 2.43 & 1.75 & 6.40 & 6.07 & 3.79 & 1.98 & 4.62 & 5.24 \\
\hline 14 & 06 Mar. 1998 & - & - & 9.97 & 8.89 & - & - & 6.01 & 6.26 & 3.96 & 2.63 & - & - \\
\hline 15 & 14 Sep. 1998 & 12.81 & 10.69 & 10.14 & 9.08 & 2.67 & 1.61 & 5.79 & 6.32 & 4.35 & 2.76 & - & - \\
\hline 16 & 10 Dec. 1998 & 12.39 & 10.70 & 9.99 & 9.12 & 2.40 & 1.58 & 5.99 & 6.39 & 4.00 & 2.73 & - & - \\
\hline 17 & 02 Sep. 1999 & 12.37 & 10.24 & 9.24 & 9.52 & 3.13 & 1.22 & - & - & - & - & - & - \\
\hline 18 & 18 Sep. 1999 & - & - & 9.59 & 9.43 & - & - & 5.46 & 6.36 & 4.13 & 3.07 & - & - \\
\hline 19 & 08 Oct. 1999 & - & - & 9.31 & 9.31 & - & - & 5.41 & 6.16 & 3.90 & 3.15 & - & - \\
\hline 20 & 03 Nov. 1999 & - & - & 8.80 & 9.25 & - & - & 4.78 & 6.17 & 4.02 & 3.74 & - & - \\
\hline 21 & 06 Dec. 1999 & 13.52 & 10.63 & 8.99 & 8.88 & 4.61 & 1.75 & 5.23 & 6.40 & 3.58 & 2.48 & 4.07 & 5.49 \\
\hline 22 & 26 Jan. 2000 & - & - & 8.89 & 8.79 & - & - & 5.15 & 6.19 & 3.74 & 2.60 & - & - \\
\hline 23 & 07 Mar. 2000 & - & - & 8.66 & 8.61 & - & - & 4.91 & 6.13 & 3.75 & 2.48 & - & - \\
\hline
\end{tabular}
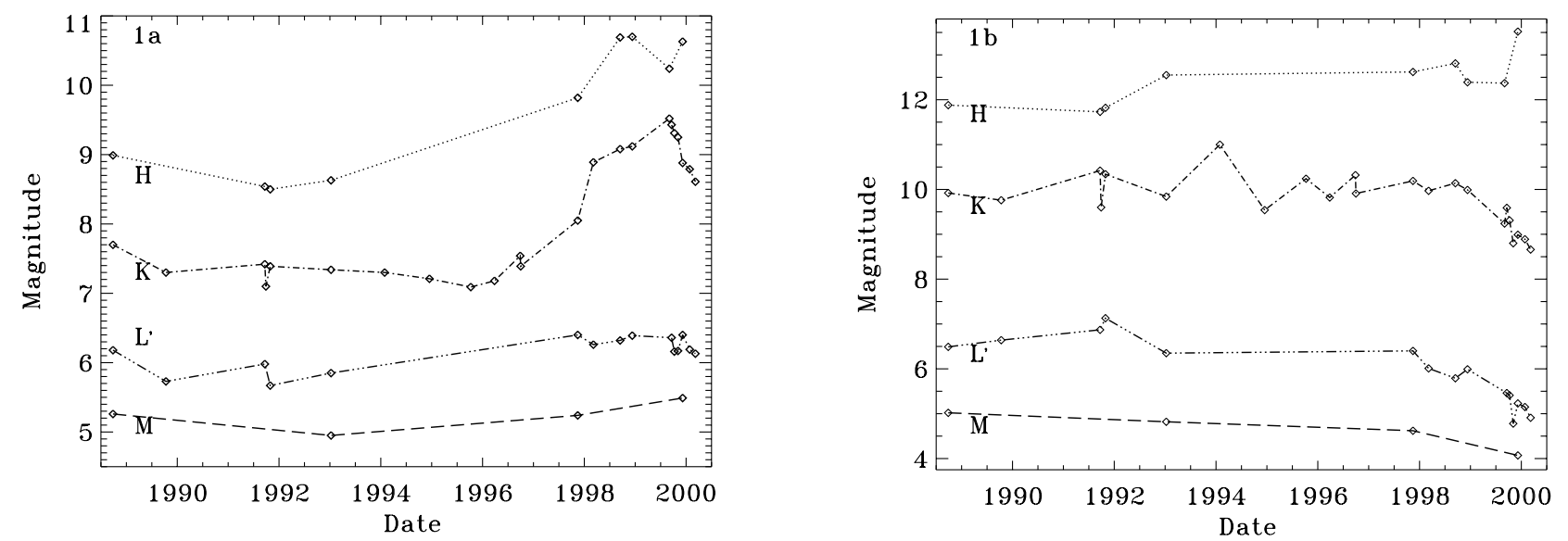

Fig. 1. Variation of the near-infrared magnitude of Haro 6-10S a) and Haro 6-10 IRC b) from 1988 to 2000

became fainter with time (Figs. 1a and b). In 1999, however, the IRC brightened, while Haro 6-10S was in a phase of decreasing brightness, so that in November the IRC was about $50 \%$ brighter than Haro $6-10 \mathrm{~S}$ at $K$. This is the first time in our experience that an IRC has been brighter at $K$ than the component seen in visible light.

Figure 2 plots the 15 available measurements of the $K-L^{\prime}$ color vs. $K$ band magnitude of Haro $6-10$ S. The figure shows that when Haro $6-10 \mathrm{~S}$ is fainter at $K$, it is also redder. The dependence of its $H-K$ color on $K$ magnitude shows a similar correlation. This has a natural explanation if changes in absorbing material along the line of sight are primarily responsible for the variation in its near infrared flux. The temporal evolution of $K-L^{\prime}$ vs. $K$ was such that the observed values lay in the lower right part of the diagram before 1997, moved upwards to the left until November 1999, and again moved downwards toward the right starting in December, 1999. This coordinated 


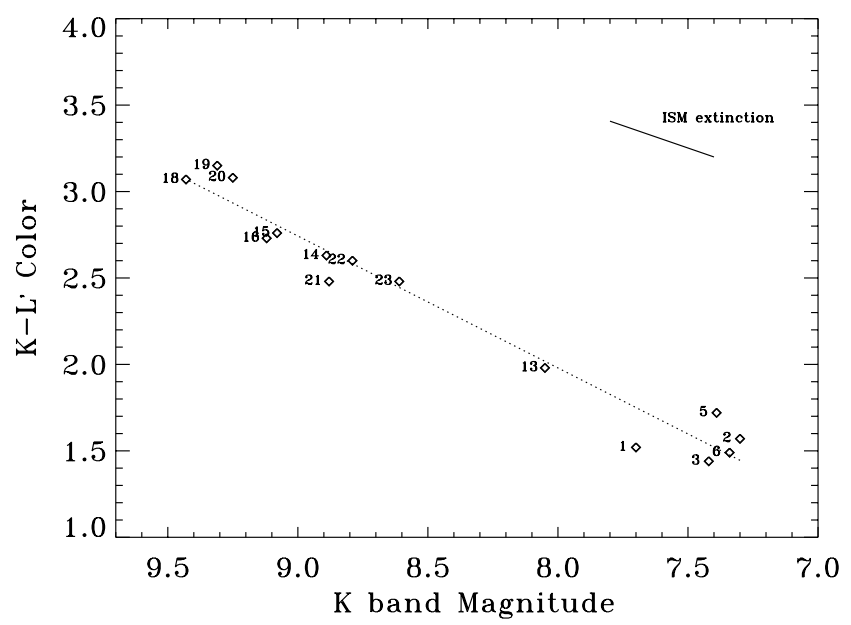

Fig. 2. $K-L^{\prime}$ color vs. $K$-band magnitude for Haro 6-10S. Overplotted on the figure is a linear fit to the data (dotted line) and the expected slope if changes in near-infrared flux are caused by variable extinction (solid line). To help identify the observation times, we identified the data points by the numbering introduced in the first column of Table 2

behavior reinforces the suggestion that a one-parameter effect, probably variable extinction, is responsible for the observed changes in flux.

Figure 2 shows the best linear fit, in the least squares sense, to the observed correlation; its slope is $0.81 \pm 0.23$. The slope for extinction following the wavelength dependence measured in the interstellar medium, $A_{K} / A_{V}=$ 0.112 and $A_{L} / A_{V}=0.058$ (Rieke \& Lebofsky 1985), is 0.51. Overplotted in Fig. 2 is a line of this slope. The difference in slopes could be attributable to a different extinction law for material around Haro 6-10S, but further multi-wavelength observations are necessary to determine if this difference is statistically significant.

Figure 3a shows $K-L^{\prime}$ vs. $K$ mag for the IRC with the data identified as in Fig. 2. The figure is divided into quadrants by lines at $K-L^{\prime}=3.55$ and $K=9.7 \mathrm{mag}$. All of the measurements before 1993 lie in the lower left quadrant, all of the $1997 / 1998$ measurements in the upper left quadrant, and the 1999/2000 measurements in the upper right corner, with the points moving right. The IRC became redder while staying faint between 1993 and 1997, and grew brighter at approximately the same colour from 1998 on. The net effect is that the IRC becam redder and brighter, which is the opposite what would be expected to result from variable extinction. This behavior is even more pronounced in a plot of the $H-K$ color vs. $K$ (Fig. 3b). Plotting $H-K$ vs. $H$ (Fig. 3c) shows a more normal behaviour in that the reddening was accompanied by an overall dimming at $H$. But here the slope of the observed $H-K$ vs. $H$ relation, $\approx 1.2$, is much too steep to be explained by extinction alone.

The Haro 6-10 binary has now been observed in the IR for more than a quarter century. Figure 4 shows the light curve of the total system in the $H, K, L^{\prime}$, and $M$ photometric bands. The measurements before 1988 are of the total, unresolved system; those before 1978 are from Elias (1978), those for 1981 from Cohen \& Schwartz (1983), and for 1984 from Myers et al. (1987). For the later data, the values are the sums of the component entries in Table 2. C. Koresko and A. Richichi (priv. comm.) provided the values for 1992 and additional data for September 1996 . The decrease of total $K$-band flux reported by Elias (1978) was accompanied by reddening of the $(H-K)$ color. This suggests that Haro 6-10S dominated the NIR flux of the system then, as it did during most of our observations, and that increased obscuration was responsible for its dimming. If we assume that indeed Haro 6-10S dominated the light of the system at $H$ and $K$ during the entire time before 1988 when only integrated measurements were available, then Haro 6-10S decreased in $K$-band flux by about a factor of 10 over the past 25 years, with major events, causing decreases by a factor of about three, during the first two and the last two years of this period. In contrast, the $L^{\prime}$ and $M$ bands dominated by the IRC show a gradual increase over the period of observations and a particularly strong increase during the past two years. This different behaviour of the light curve at short and long wavelengths also suggests that different physical phenomena are responsible for the variability of Haro 6-10S and the IRC.

\subsection{Ice-band variability}

Figures $5 \mathrm{a}$ and $5 \mathrm{~b}$ show measurements of spectral energy distributions of Haro 6-10S and the IRC centered on the $3.1 \mu \mathrm{m}$ water-ice feature. We estimated the ice-band optical depth, $\tau_{\text {ice }}$, by measuring the absorption with respect to a continuum that was drawn as a straight-line fit between the fluxes at $K$ and $L^{\prime}$ (or $3.5 \mu \mathrm{m}$ if no $L^{\prime}$ measurement was available). These values, and their uncertainties, are indicated in Figs. 5a and 5b. The uncertainties in these estimates are the result of the variable shape of the component SEDs, incomplete spectral sampling of the SEDs, and imprecision of the photometry. We find that the optical depths to Haro 6-10S and its IRC are variable at the $3.5 \sigma$ and $2 \sigma$ levels, respectively.

The large uncertainties in the values of $\tau_{\text {ice }}$ make it difficult to establish correlations. However, for Haro 6-10S the decrease in $K$ brightness after 1997 by about 1.5 magnitudes was accompanied by an increase of $\tau_{\text {ice }}$ by about 0.3 . If the brightness decrease is due to increased extinction as we have argued in Sect. 3.1, then this increase in $\tau_{\text {ice }}$ is much less than the change in optical depth of the ice band by 1.2 expected from Whittet et al. (1988) relation between $A_{V}$ and $\tau_{\text {ice }}$ for the interstellar medium (ISM). This suggests again that the material around Haro 6-10 may have properties different from the general ISM. For the IRC no clear conclusions can be drawn, but high values of $\tau_{\text {ice }}$ did not occur in our measurements when the IRC was brighter than $K=9$ mag.

It is interesting to consider the average $\tau_{\text {ice }}$ toward Haro 6-10S and the IRC, which have values of $0.34 \pm 0.06$ 

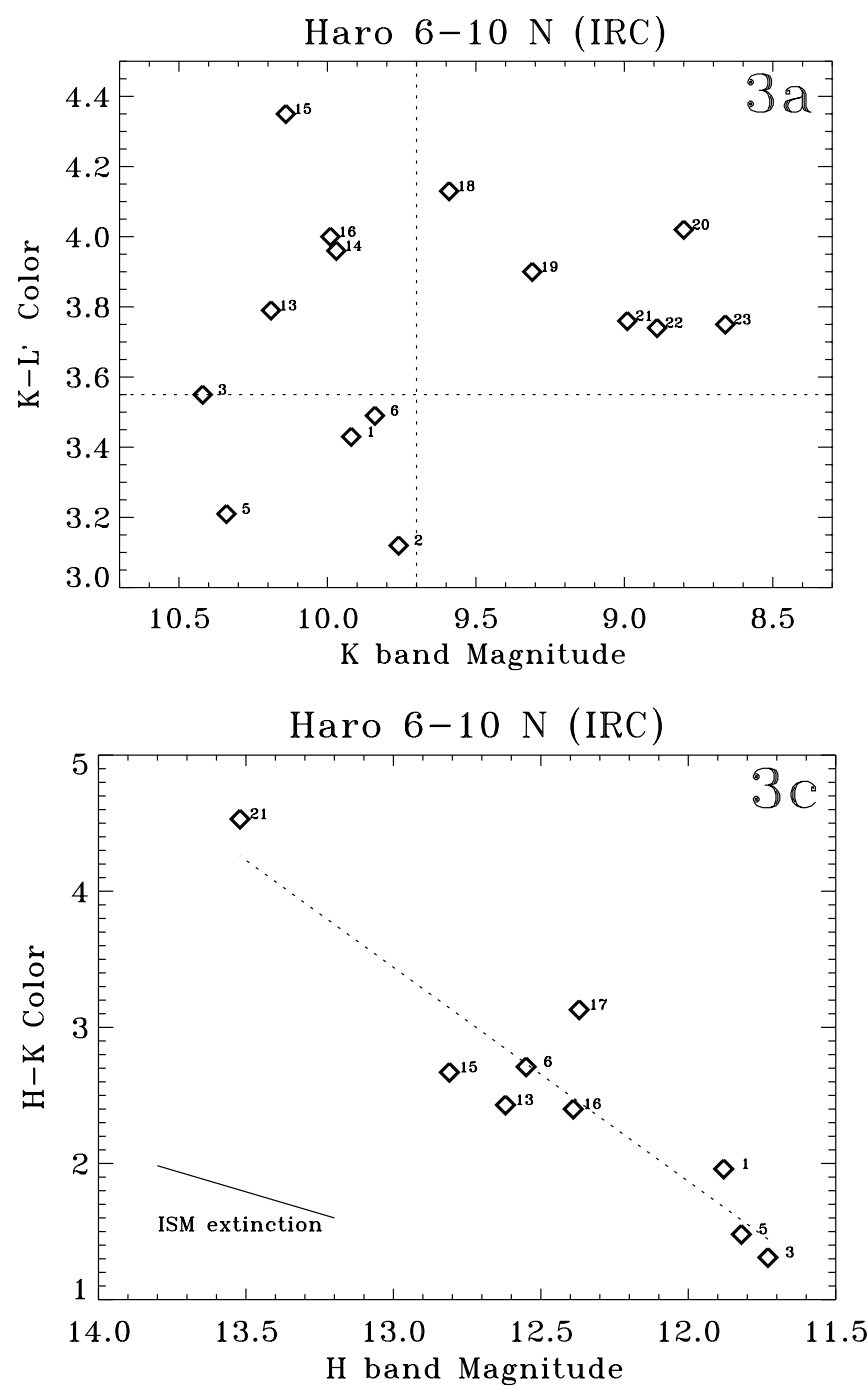

and $0.68 \pm 0.07$, respectively. Using the $\tau_{\text {ice }}$ to $A_{V}$ relation from Whittet et al. (1988), $\tau_{\text {ice }}=m\left(A_{V}-A_{V}(0)\right)$ where $A_{V}(0)=3.3 \pm 0.1$ and $m=0.093 \pm 0.003$, we derive average visual extinctions $7.0 \pm 0.7 \mathrm{mag}$ for Haro 6-10S, and $10.6 \pm 0.8$ for the IRC. Our result is consistent with previous angularly resolved observations of the silicate and water-ice absorption features (van Cleve et al. 1994; Leinert et al. 1996).

\subsection{Morphology in the near infrared}

In December 1999 we imaged Haro 6-10 at UKIRT using the near-IR camera TUFTI. It is equipped with a $256 \times$ 256 pixel $^{2}$ InSb detector. The optics provide a plate scale of $0.081 \mathrm{arcsec} / \mathrm{pixel}$ to take advantage of the wavefront sensing and active optics capabilities of the UKIRT.

Figure 6 shows the images obtained in the $J, H, K$, "ice" $(3.10 \mu \mathrm{m})$, "dust" $(3.28 \mu \mathrm{m}), L^{\prime}$, and $M$ filters. Haro 6 -10S and the IRC are resolved in all the images except $J$ in which the IRC was not detected. At the time of these observations the components were essentially equal in brightness at $K$ and the IRC was brighter at longer wavelengths (see also Table 2). The sensitivity of the $K$

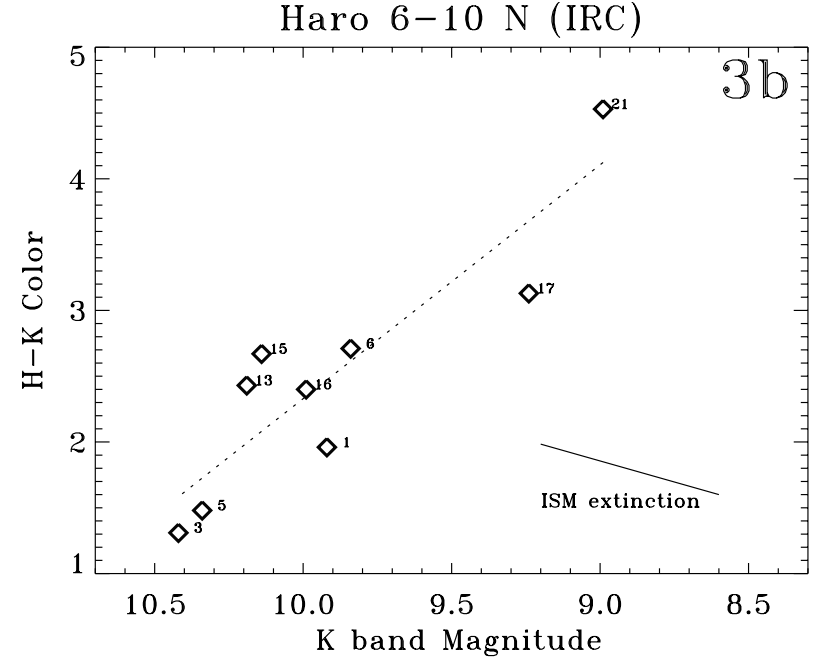

Fig. 3. a) presents the $K-L^{\prime}$ color vs. $K$-band magnitude for the Haro 6-10 IRC. b) is the $H-K$ color vs. $K$-band magnitude plot and c) is the $H-K$ color vs. $H$-band magnitude. Overplotted on $\mathbf{b}$ ) and $\mathbf{c}$ ) is a linear fit to the data (dotted line) and the expected slope if near infrared flux variation is caused by variable extinction (solid line). In the three plots, the data points are numbered by observation date as they appear in Table 2 . We note that in a) the lower left quadrant contains data from before 1993, the upper left quadrant data from $1997 / 98$, and the upper right quadrant data from 1999/2000

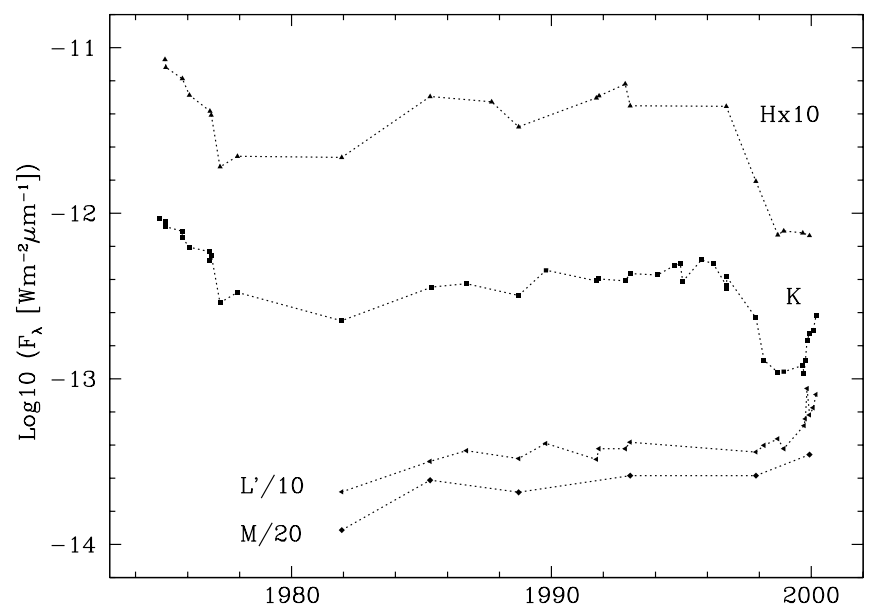

Fig. 4. Variation of near-infrared flux of Haro 6-10 from 1974 to 2000. Measurements before 1978 are from Elias (1978), for 1981 from Cohen \& Schwartz (1983), for 1984 from Myers et al. (1987), for November 1992 and September 1996 from C. Koresko and A. Richichi (private communications)

frames is insufficient to show the faint north-south extensions on the IRC found by Koresko et al. (1999). The short 

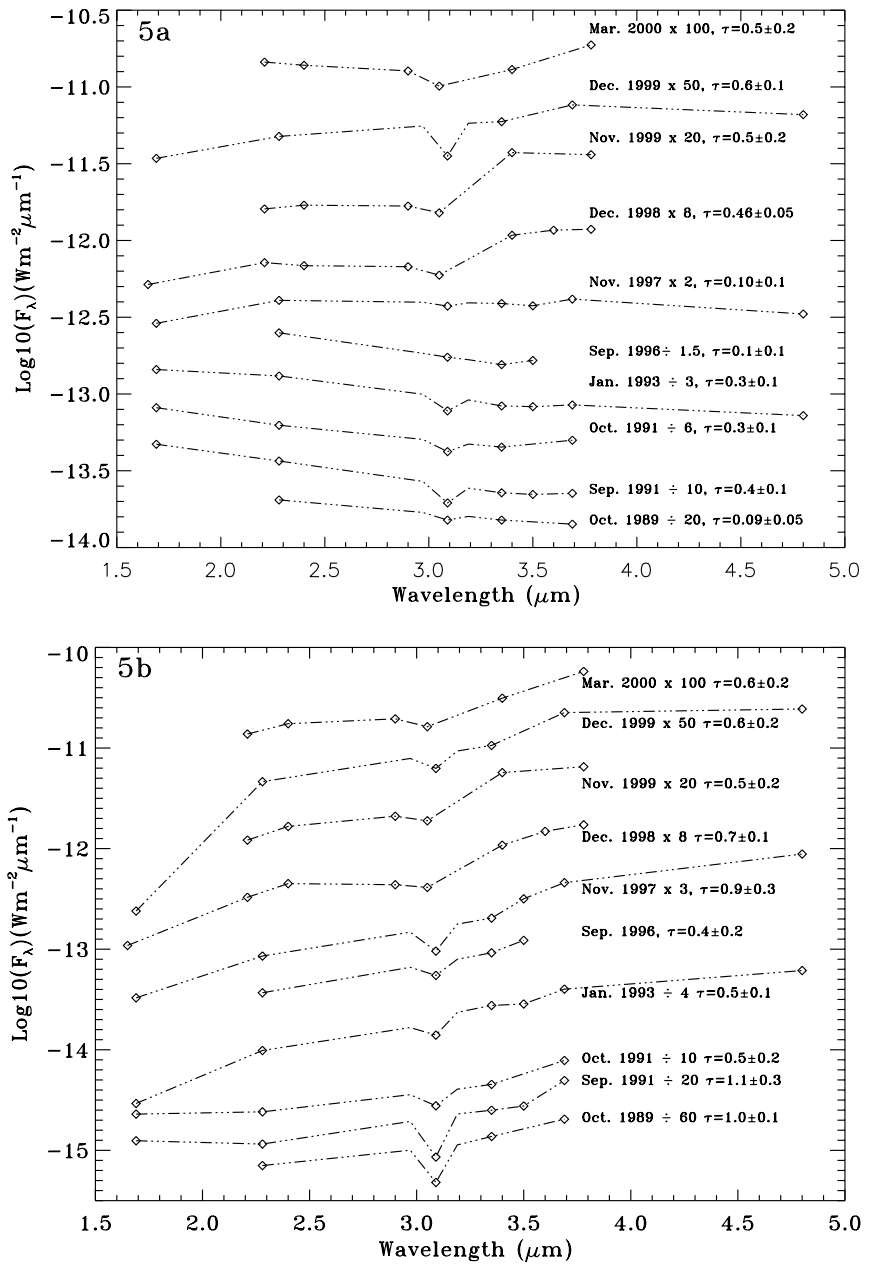

Fig. 5. Variation in ice-band absorption of Haro 6-10S a) and its IRC $\mathbf{b}$ )

wavelength images show the jetlike structure toward the south discovered by Movsessian \& Magakian (1999) in the light of the S[II] $6716+6731$ line emission and imaged in $K$ by Koresko et al. (Koresko 1999). We emphasize this structure in $J$ and $H$ images by suitable choice of the scaling. Obviously, scattered light contributes strongly to the brightness of this feature and its classification as collimated supersonic outflow therefore requires further study. Another interesting feature, best visible at $H$, is an arc of emission $6.2 \mathrm{E}$ of the binary system, extending roughly from position angle $90^{\circ}$ to $120^{\circ}$. The arc, marginally visible on the image of Koresko et al. (1999), is apparently connected to the nebulosity closer to the system both at its northern and southern end. Spectral line imaging is necessary to clarify if this is another bow shock, similar to $\mathrm{HH} 184 \mathrm{~F}$ and $\mathrm{G}$ shown in the paper of Devine et al. (1999). If it is a bow shock, then it defines a new outflow system, at $\mathrm{PA} \approx 110^{\circ}$ and adds one more complexity to the immediate environment of Haro 6-10. At longer wavelengths ( $L^{\prime}$ and $M$ filters), emission from the infrared companion becomes dominant with respect to the southern component and the jetlike feature is no longer detected. Despite the substantial brightness changes of the
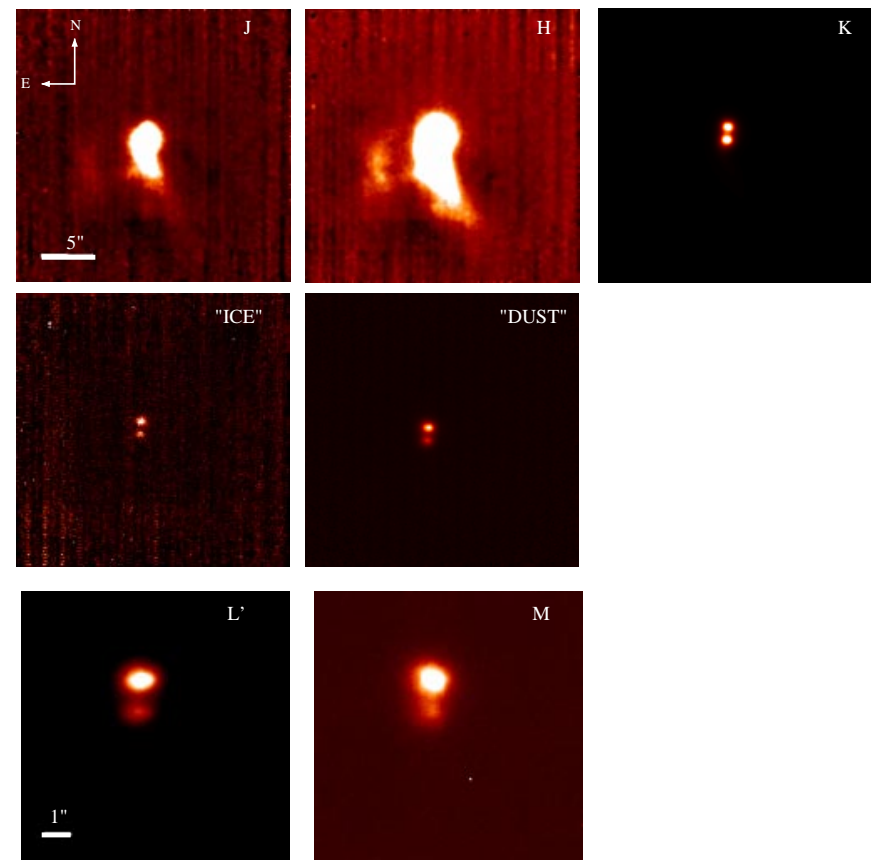

Fig. 6. Images of Haro 6-10 and its companion obtained in different near-infrared bands from UKIRT in December 1999. The images in $J, H, K$, "ice", and "dust" filters are a mosaic of 5 dithered images. The images in $L^{\prime}$ and $M$ filters represents a single frame. The different scales are shown in the $J$ and in the $L^{\prime}$ images

components of Haro 6-10 there were no significant changes in morphology.

\section{Discussion}

Our results show convincingly, by the near quantitative agreement with the reddening predictions for such a model, that the NIR flux variability of the star seen in visible light, Haro 6-10S, is caused in large part by variations in extinction. The average extinction measured towards Haro 6-10S using the ice-band is in good agreement with K97's estimate, $A_{V}=5.6 \mathrm{mag}$, if we allow for the fact that our observations included a period of higher extinction. Our data do not provide information directly about where the obscuring material lies and why its column density varies. The variability is probably attributable to inhomogeneities in the obscuring material and their motion across our line of sight. Since this line of sight contains sufficient material to produce $A_{V}=5$ to $10 \mathrm{mag}$, but yet is sufficiently transparent to permit detection of the star, it may prove useful for a comparison of the composition of material near a young star with that in the interstellar medium. For example, it should be possible to compare the ratios of the water ice and silicates in the two environments.

Changes in accretion rate do not appear to be a main cause for the brightness variations of Haro 6-10S. From the example given by Calvet et al. (1997) we find the near-infrared colours $H-K$ and $K-L^{\prime}$ almost 
independent of accretion rate over the range of $10^{-8} M_{\odot} /$ yr to $10^{-6} M_{\odot} /$ yr. Emission from an infalling envelope, as probably present in highly veiled sources, tends to redden the energy distribution with increasing accretion luminosity, which is not what we observe. Quantitative predictions of these intricate effects would need specific modelling of the source and are beyond the scope of our paper.

The flux variability of the IRC is much more complex than that of Haro 6-10S. The extinction implied by the ice-band absorption is much smaller than K97's estimate of $A_{V} \approx 49$ mag derived based on the assumption that the IRC is a strongly obscured young star. A certain discrepancy in the derived $A_{V}$ is not too surprising because the relatively large value of $\tau_{\text {ice }}$ suggests that the line of sight could be optically thick in the ice-band. Radiative transfer effects and scattering could then produce an underestimate of the actual extinction to a star powering the IRC. But more significantly, the color variations of the IRC (e.g. Fig. 3) indicate that processes other than, or in addition to, extinction must be involved in its variability. Interpretation of reddening by extinction therefore may not be adequate for Haro 6-10N. Herbst et al.'s (1995) detection of $\mathrm{H}_{2}$ toward the IRC but not Haro 6-10S indicates the presence of shocked material, which points to accretion as an additional process playing a role in the IRC variations. Muzzerole et al. (1998) have demonstrated the usefulness of $\mathrm{Br} \gamma$ emission to measure the accretion luminosity. A time series of angularly resolved measurements of $H_{2}, \operatorname{Br} \gamma$ and $\operatorname{Br} \alpha$ emission of the IRC is likely to provide a useful diagnostic of its activity and provide further insights to its nature, in particular when coupled to monitoring of extinction indicators such as reddening and dust absorption features.

\section{Summary}

Our angularly resolved NIR observations of the Haro 6-10 binary show that:

1. Both components vary significantly in near infrared flux on timescales of a few months;

2. The near infrared variability of Haro 6-10S, the visible light star, is caused mostly by changes in extinction. On the other hand, the near infrared variability of the IRC cannot be explained solely by variable extinction;

3. Absorption by water-ice is present in both components and is consistently stronger toward the IRC. There is evidence that the ice-band absorption is variable in time toward both components.
Acknowledgements. We acknowledge with pleasure the hospitality provided by Hans Zinnecker and the organizers of IAU Symposium 2000 where this paper was conceived. We thank C. Koresko and A. Richichi for allowing us to use their 1992 and September 1996 data, and D. Griep and P. FukumuraSawada for obtaining the service observations at the IRTF in March 2000. The work of TB and MS was supported in part by NSF Grant AST98-19694.

\section{References}

Calvet, N., Hartmann, L., \& Strom, S. E. 1997, ApJ, 481, 912 van Cleve, J. E., Hayward, T. L., Miles, J. W., et al. 1994, Ap\&SS, 212, 231

Cohen, M., \& Schwartz, R. D. 1983, ApJ, 265, 877

Devine, D., Reipurth, B., Bally, J., \& Balonek, T. J. 1999, AJ, 117,2931

Elias, J. H. 1978, ApJ, 224, 857

Goodrich, R. W. 1986, AJ, 92, 885

Herbst, T. M., Koresko, C. D., \& Leinert, Ch. 1995, ApJ, 444, L93

Köhler, R., Kunkel, M., Leinert, Ch., \& Zinnecker, H. 2000, A\&A, 356, 541

Koresko, C. D., Herbst, T. M., \& Leinert, Ch. 1997, ApJ, 480, 741

Koresko, C. D., Blake, G. A., \& Brown, M. E. 1999, ApJ, 525, L49

Lada, C. J. 1987, in Star Forming Regions, proceedings of IAU Symposium No. 115, ed. M. Peimbert, \& J. Juguka (Reidel, Dordrecht), 1

Leinert, Ch., \& Haas, M. 1989a, ApJ, 342, L39

Leinert, Ch., \& Haas, M. 1989b, A\&A, 221, 110

Leinert, Ch., Haas, M., \& Weitzel, N. 1996, in Disks and Outflows around Young Stars, ed. S. V. W. Beckwith, J. Staude, A. Quetz, \& A. Natta, Lecture Notes in Physics, 465 (Springer Berlin, CD-ROM), 411

Movsessian, T. A., \& Magakian, T. Yu. 1999, A\&A, 347, 266

Myers, P. C., Fuller, G. A., Mathieu, R. D., et al. 1987, ApJ, 319,340

Rieke, G. H., \& Lebofsky, M. J. 1985, ApJ, 288, 618

Richichi, A., Leinert, Ch., Jameson, R., \& Zinnecker, H. 1994, A\&A, 287, 145

Sato, S., Nagata, T., Tanaka, M., \& Yamamoto, T. 1990, ApJ, 359, 192

Strom, K. M., Strom, S. E., Wolff, S. C., Morgan, J., \& Wenz, M. 1986, ApJS, 62, 39

Tokunaga, A. T. 1999, in Allen's Astrophysical Quantities, fourth edition, ed. A. N. Cox (Springer New York/Berlin/Heidelberg), 150

Whittet, D. C. B., Longmore, A. J., \& McFadzean, A. D. 1985, MNRAS, 216, 45

Whittet, D. C. B., Bode, M. F., Longmore, A.J., et al. 1988, MNRAS, 233, 321 